\title{
Nonfrustrated Antiferromagnet in a Frustrated Lattice Due to Charge Ordering: A Scenario for Pairing in Layered Cobaltates
}

\author{
P. WróBel ${ }^{a, b}$ AND W. SUleja ${ }^{a}$ \\ ${ }^{a}$ Institute of Low Temperature and Structure Research \\ P.O. Box 1410, 50-950 Wrocław 2, Poland \\ ${ }^{b}$ Max Planck Institute for the Physics of Complex Systems \\ Nöthnitzer Str. 38, 01187 Dresden, Germany
}

Dedicated to Professor Józef Spatek on the occasion of his 60th birthday

The mechanism of pairing-symmetry selection in the weakly electron doped $t-J$ model on the honeycomb lattice has been analyzed. The discussion of that problem has been motivated by some recent suggestions that due to charge ordering which may take place in the unconventional superconductor $\mathrm{Na}_{x} \mathrm{CoO}_{2} \cdot y \mathrm{H}_{2} \mathrm{O}$ at doping levels near $x=1 / 3$ the physics of $\mathrm{CoO}_{2}$ planes may be effectively described in terms of a model for a weakly electron doped antiferromagnet on the honeycomb lattice. In the current publication the main emphasis has been put on reviewing experimental and theoretical work, the results of which support the scenario of charge order. In the calculation, the so-called string picture has been used. It has been demonstrated that spin fluctuations may induce in the honeycomb lattice the formation of an unconventional two-particle bound state. Upon the condensation of bound particles this mechanism may give rise to unconventional pairing. The critical value of the ratio $J / t$ which is sufficient to induce binding has not been evaluated. It has been assumed instead that in the case of cobaltates some additional isotropic attractive interaction, for example phonon mediated, is active. Despite that the exchange of spin fluctuations is not a dominating interaction, it selects the symmetry of the paired state because it is anisotropic. $C_{3 v}$ is the relevant point group for the $t-J$ model on the honeycomb lattice. It has been shown that the bound state of two additional electrons doped to the half-filled antiferromagnetically ordered system has zero total momentum and $p$-wave symmetry of the irreducible representation $E$. The expected paired state is a mixture of a singlet and a triplet because the honeycomb lattice does not possess the inversion symmetry.

PACS numbers: 71.10.Fd, 74.20.Mn, 75.50.Ee 


\section{Introduction}

A lot of attention has been recently paid to the discovery of superconductivity (SC) in a hydrated sodium cobaltate $\mathrm{Na}_{x} \mathrm{CoO}_{2} \cdot y \mathrm{H}_{2} \mathrm{O}$ [1]. Cobaltates are the second class of layered $3 d$ transition-metal oxides in which this phenomenon has been observed. Similar materials in which this phenomenon has been discovered first are cuprates. The main difference between those two groups of compounds is the crystallographic structure of a layer. In cuprates, $\mathrm{Cu}$ ions form the square lattice in a single layer, while in cobaltates, Co ions form the triangular lattice. Triplet pairing has been suggested in order to interpret results of some experiments [2-5] on cobaltates. Contradicting conclusions have been drawn after the analysis of some other experiments $[6,7]$, namely that singlet pairing is generated. Nevertheless, there is no doubt about the fact that pairing in layered cobalt oxides is unconventional $[8,9]$. Many different suggestions based on theoretical analyses have been made in order to elucidate the mechanism of SC in those systems [10-19], but no final conclusions regarding the symmetry and the total spin of the paired state have been drawn. Surprisingly, even before the discovery of superconductivity in hydrated cobaltates a theoretical analysis, which may be relevant to that phenomenon, has been performed [20]. It concerned the $t-J$ model $(t J \mathrm{M})$ on the triangular lattice. Much later it has been suggested that the electron doped $t-J$ model on the triangular lattice formed by cobalt ions is indeed suitable for analyzing them [10].

\section{Review of theoretical and experimental results suggesting charge ordering in cobaltates at the doping level $x=1 / 3$}

The formation of spin and charge ordering in $\mathrm{Na}_{x} \mathrm{CoO}_{2}$ at the doping level near $x=1 / 3$ has been recently suggested [21-24]. The appearance of SC which occurs in the range $0.22 \leq x \leq 0.47$ may be related to that ordering. Some experimental results also indicate that the scenario of charge modulations may be realized in cobaltates for $x=1 / 3$. For example, it has been demonstrated by means of angle-resolved photoelectron spectroscopy (ARPES) [25] that in this case all parts of the Fermi surface have the nesting property for some fundamental reciprocal lattice vectors which are related with a well-defined superlattice structure. The results of these measurements indicate that cobaltates are even more unconventional superconductors than cuprates. For example in the case of cobaltates the Fermi velocity is even smaller than in the case of cuprates and much smaller than in the case of conventional superconductors. Similarly, the ratio of the Fermi energy to the energy of optic phonons and the calculated phase ordering temperature are also smaller, while the effective mass is much bigger. Only the calculated size of the pair wave function is comparable to that of cuprates. The observed nesting property can lead to a hypothesis that in the charge ordered state exactly at $x=1 / 3$, cobalt atoms which are in the valence state $\mathrm{Co}^{3+}$ and in the spin-0 state form a new triangular lattice with a larger elementary cell. In the language 
of the electron-doped $t J \mathrm{M}$ those sites are doubly occupied and therefore they do not influence magnetic and transport properties of the system at the doping level $x=1 / 3$ and slightly above that value. The honeycomb lattice is formed by the rest of cobalt atoms which are in the valence state $\mathrm{Co}^{3+}$ and in the spin- $\frac{1}{2}$ state. Some sites which belong to that newly effectively formed honeycomb lattice will become doubly occupied upon additional doping. Thus, we can draw a conclusion that the analysis of the $t J \mathrm{M}$ model on the honeycomb lattice in the limit of weak electron doping may give some insight into the physics of SC cobaltates for doping levels slightly higher than $x=1 / 3$.

Now we proceed to analyze in more detail theory-based arguments in favor of the charge ordering scenario for the doping level $x=1 / 3$. Motrunich and Lee [21] have suggested that charge frustration due to a further neighbor Coulomb repulsion can influence the electronic properties of $\mathrm{Na}_{x} \mathrm{CoO}_{2}$ in the full doping range. It can result in the reduction of charge mobility and the decrease in Fermi energy which sets the scale of the degeneracy temperature. Due to the decrease in the degeneracy temperature the system may behave in an unconventional way in comparison with standard metals, which is indeed observed in the case of cobaltates. At the commensurate filling $1 / 3$ the tendency towards charge ordering due to the long-range Coulomb interaction should be particularly strong. In the framework of the variational analysis for the Jastrow-Gutzwiller wave function these authors have found that the $t J \mathrm{M}$ supplemented by the additional nearest-neighbor (NN) repulsive interaction reveals at the doping level $1 / 3$ a $\sqrt{3} \times \sqrt{3}$ charge order of the form which underlies the scenario on which the analysis presented here is based.

The local density approach in the form which allows us to take into account the role of the Coulomb interaction (local density approximation, LDA+U) has been also used by Lee et al. to analyze tendencies towards charge ordering in doped cobaltates [22]. These authors have shown that even for moderate correlation strength charge ordering and antiferromagnetism in the form which has been assumed here are favored. Their results also suggest only a small enhancement of linear specific heat and itinerant character of magnetism, which agrees with experimental data.

Zheng et al. have applied a series expansion and a cluster mean field approach to discuss the behavior of cobaltates in the framework of the $t J \mathrm{M}$ supplemented by the NN Coulomb repulsion [23]. The results of their analysis are also in favor of the charge ordering scenario which underlies further calculations presented here. Another interesting conclusion which can be drawn from the work by Zheng and collaborators is that NN repulsion excludes the possibility of phase separation which is expected to appear in the bare $t J \mathrm{M}$.

Motivated by the hypothesis of charge ordering in cobaltates, in the following part of the paper we will analyze a pairing scenario which is based on that hypothesis. 


\section{Spin bipolaron formation in the antiferromagnetic on the honeycomb lattice}

Since the honeycomb lattice is bipartite, by performing the particle-hole transformation and by changing additionally the sign of fermion operators for sites which belong to one of sublattices one can prove that the $t J \mathrm{M}$ on that lattice is invariant with respect to the particle-hole transformation for the filling of one electron per site. Therefore, it is in principle possible to analyze the equivalent hole doped version of that model with the same number of holes created in the half filled system as the number of additional electrons in the relevant electron doped version of it. We will indeed perform the calculation for the hole doped system. In the case of cobaltates the condition $|t| \gg J$ is obeyed and $t$ is negative.

Our main goal is to understand the mechanism of pairing-symmetry selection in the cobaltates in the framework of the spin polaron approach applied to the hole doped $t J \mathrm{M}$ on the honeycomb lattice. We will apply the spin bipolaron (SBP) approach which is a most basic version of this method. This approach is based on the assumption that antiferromagnetic (AF) spin fluctuations give rise to the formation a tightly bound two-hole state and on the assumption that the problem of two holes in the honeycomb lattice may be reduced to the analysis of hopping performed by a single boson which represents a bound hole pair. We will not attempt to find out for what critical value of the ratio $J / t$ hole binding starts to take place on the honeycomb lattice. This parameter can be found by means of a calculation performed in the framework of a different method, as for example by means of an exact diagonalization, which is beyond the scope of this paper. Furthermore, it is not clear if electron correlations alone can drive pairing in cobaltates or if a different source of attraction, as for example phonon exchange, is involved in this process. If the supplementary moderate effective attraction in the form of a density-density interaction is isotropic, it can be expected that the spin fluctuation mechanism will still decide about the symmetry of the paired state.

The SBP approach is based on the following scenario. We assume that at least short-range AF correlations exist in the weakly doped $t J \mathrm{M}$ on the honeycomb lattice because this lattice is bipartite. We make this assumption while having in mind that, according to our hypothesis, the cobalt oxide plane may be modeled for doping levels slightly above the value $1 / 3$ by the weakly electron-doped $t J \mathrm{M}$ on the honeycomb lattice.

Hopping of holes in the locally AF spin background is accompanied by shifts of spins and creation of defects in that background, which gives rise to the increase in the exchange energy, due to the formation of "ferromagnetic" bonds at NN sites. This process also brings about temporal confinement of holes by the defects which can be also interpreted as fluctuations of the AF background.

The fluctuations form chains which lie on paths along which holes have traveled. Chains act on holes as strings because the exchange energy grows linearly 
with the number of fluctuations. Therefore we assume that only short chains should be considered in detail, while details regarding longer strings can be neglected. If the hole moves back along the same path towards the initial site where it has been created a string-like chain of defects is shortened. Since charge dynamics is much faster than spin dynamics for $|t| \gg J$, the retraceable hopping of a hole forth and back along a chain plays an important role in determining system properties. The properties of the low energy sector in the Hilbert space of a weakly doped AF are related to the process of string formation. Within the SBP approach that sector consists of SBP states representing holes quasi-confined by strings in regions around some pairs of sites where holes have been initially created. These states are ground states of a trial "unperturbed Hamiltonian" which by construction does not contain matrix elements that give rise to deconfinement of holes.

At the next stage of the analysis, after SBPs have been constructed processes which give rise to hole deconfinement are considered. They are often related to some path details neglected during the construction of spin polarons. These processes determine the coupling between SBPs and the form of an effective Hamiltonian represented in the basis of SBP states.

Now we proceed to present the SBP construction and the mechanism of hole pair propagation with some detail. The propagation of two holes together is easier than the motion of a single hole. As we have already mentioned the object which propagates is actually more complex than just a bare hole pair. It can be identified as an AF SBP because a deformation of the spin background accompanies it. An SBP wave function $\left|\Psi_{\langle i, j\rangle}\right\rangle$ can be defined as a combination of states which may be created by independent hopping of holes created at a pair of NN sites $\langle i, j\rangle$,

$$
\left|\Psi_{\langle i, j\rangle}\right\rangle=\sum_{\mathcal{P}_{i}, \mathcal{P}_{j}} \alpha_{\mathcal{P}_{i}, \mathcal{P}_{j}}\left|\mathcal{P}_{i}, \mathcal{P}_{j}\right\rangle .
$$

In Fig. 1a, b, c, and e examples of such states have been shown. The zero-length string-state representing two holes created in the Néel state at NN sites also contributes to the superposition defining the SBP wave function. According to the convention applied by us, hole hopping which creates new components of the SBP wave function is induced by operators in the form $c_{i, \sigma} c_{j, \sigma}^{\dagger}$, where $i$ and $j$ are NN sites. $\mathcal{P}_{i}$ parameterizes the geometry of a path along which the hole that starts from the site $i$ has been moving and $\left|\mathcal{P}_{i}, \mathcal{P}_{j}\right\rangle$ is a state, which has been created in this way. By definition, we prohibit, at this stage of considerations, each hole to follow the accompanying hole along the trace left by the latter. By means of that restriction we achieve that SBP states are confined in the region around the pair of sites on which a bare hole pair has been initially created, which is a property useful for constructing an effective Hamiltonian for the weakly doped $t J \mathrm{M}$ on the honeycomb lattice. We apply an approximation according to which the coefficients $\alpha_{\mathcal{P}_{i}, \mathcal{P}_{j}}$ in (1) depend only on the lengths $\mu, \nu$ of paths $\mathcal{P}_{i}$ and $\mathcal{P}_{j}$, $\alpha_{\mathcal{P}_{i}, \mathcal{P}_{j}} \equiv \alpha_{\mu, \nu}$. These coefficients are solutions of the Schrödinger equation which 

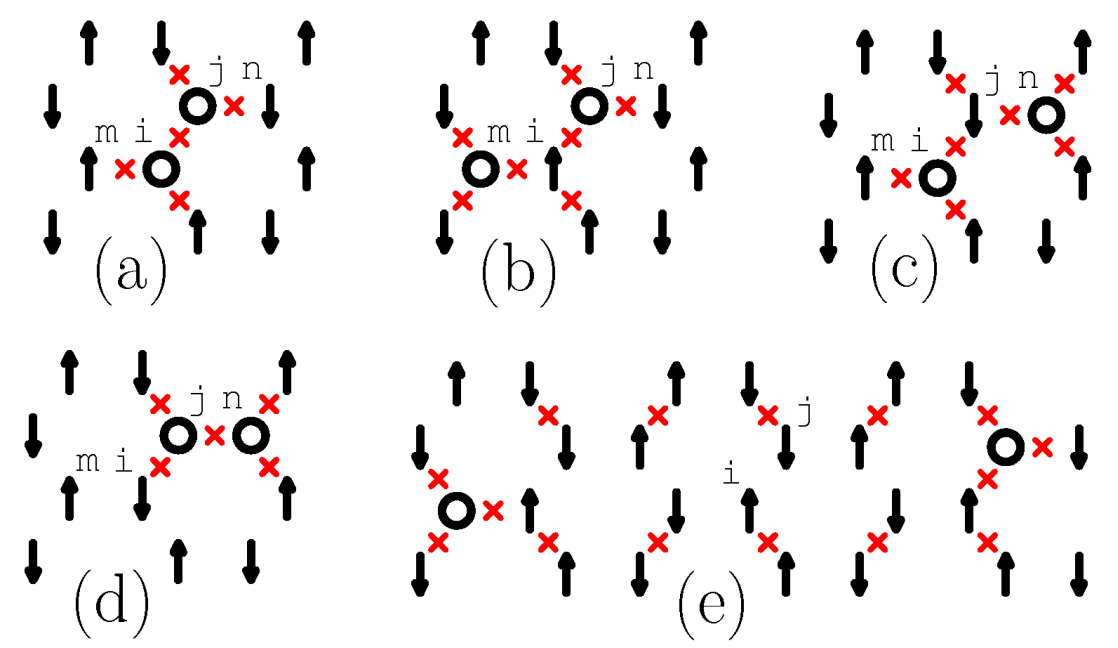

Fig. 1. Some states which contribute to the superposition defining the wave function for the SBP created at sites $i, j$ : (a), (b), (c), and (e). Also a process giving rise to the hopping of the SBP which is represented in our effective model by a hard-core boson. One of two holes created in the Néel state at sites $i, j$ (a) is being shifted from site $j$ to site $n$ (c). The move of the second hole from site $i$ to site $j$ is giving rise to a state representing two holes created at sites $j, n$ in the Néel state (d).

describes a hole pair in a potential well formed by string-like lines of defects,

$$
\begin{gathered}
t\left[\alpha_{\mu-1, \nu}+(z-1) \alpha_{\mu+1, \nu}+\alpha_{\mu, \nu-1}+(z-1) \alpha_{\mu, \nu+1}\right] \\
+\frac{J}{2}\left(6+\mu+\nu-\delta_{\mu+\nu, 0}\right) \alpha_{\mu, \nu}=E_{2} \alpha_{\mu, \nu},
\end{gathered}
$$

where $z=3$ is the coordination number. The lengths $\mu$ and $\nu$ cannot be negative, thus we also assume that $\alpha_{\mu, \nu}=0$ for $\mu<0$ or $\nu<0$. The origin of this equation which defines the SBP is easy to understand. The form of the first part on the left side follows from the fact that longer paths may be obtained from a given path by extending it during a hop in $z-1$ different directions and that there exists only one possible hole move by means of which a string may be shortened by one step. The second part counts the number of pairs of NN sites which are not occupied by anti-parallel spins. All such broken bonds raise the energy by $J / 2$ per bond in comparison to the energy of the Néel state. The factors $\alpha_{\mu, \nu}$ also obey an additional normalization condition,

$$
\sum_{\mu=0, \nu=0}(z-1)^{\mu+\nu} \alpha_{\mu, \nu}^{2}=1 .
$$

The factor $(z-1)^{\mu+\nu}$ in the above formula represents the number of different paths obtained by means of $\mu$ and $\nu$ non-retraceable hops of the first and of the second hole, respectively. 


\section{Simple description of quasiparticle interaction in doped AF on the honeycomb lattice}

Equation (2) represents a trial Hamiltonian, which does not describe all processes contained in the $t J \mathrm{M}$. We derive an effective Hamiltonian represented in terms of SBPs by analyzing matrix elements of the full $t J \mathrm{M}$ between SBP states defined as the ground state of Eq. (2). Thus, processes responsible for SBP deconfinement are also considered now. They give rise to off-diagonal terms in the effective Hamiltonian. SBPs are composite objects consisting of two quasi-confined hole-like quasiparticles. Therefore, the effective Hamiltonian describing their hopping actually represents the interaction between quasiparticles.

The mechanism of hole-pair deconfinement in the AF spin background is actually rather simple. Let us start its analysis with two holes created at NN sites $i, j$ in the honeycomb lattice, Fig. 1a. After a single hop of a hole from site $j$ to site $n$ a defect in the AF spin background on site $j$ is created, Fig. 1c, and the exchange energy increases. That defect is annihilated when the hole initially created at the site $i$ moves to the site $j$, Fig. 1 d. During this process the hole pair moves from the pair of sites $i, j$ to the pair of sites $j, n$ and no defects in the AF environment are left. The hole-pair propagation is in reality more complex because it involves not only bare holes but also the cloud of spin fluctuations around them and longer strings as one depicted in Fig. 1e, which means that an object which moves is actually an SBP. We will perform a more quantitative analysis of this process. Figures 1a and c depict string states which contribute to the SBP state $\left|\Psi_{\langle i, j\rangle}\right\rangle$ because they have been obtained by means of non-retraceable hopping of two holes which have started from sites $i, j$. The state depicted in Fig. 1d does not belong to this group. Since the state presented in Fig. 1d represents two holes created at another pair of sites $j, n$, it is a string state which is a component of another SBP state $\left|\Psi_{\langle j, n\rangle}\right\rangle$. On the other hand, the state depicted in Fig. 1d can be obtained by the action of a term in the hopping operator of the $t J \mathrm{M}$ on the state represented by Fig. 1c. As we know, the latter state is a component of the SBP $\left|\Psi_{\langle i, j\rangle}\right\rangle$ created at sites $i, j$. Within the SBP formalism this fact gives rise to the coupling of wave functions for SBPs created at different pairs of sites $i, j$ and $j, n$ by the hopping operator in the $t J \mathrm{M}$, and to a nonzero contribution to the matrix element $\left\langle\Psi_{\langle i, j\rangle}|H| \Psi_{\langle j, n\rangle}\right\rangle$. Longer strings which start at site $i$ and lead through sites $j$, and $n$, but do not end at the latter site also contribute to the coupling between wave functions of SBPs formed on pairs of sites $i, j$ and $j, n$. These strings may be obtained by shifting in the non-retraceable way the hole which occupies the site $n$ in Fig. 1c. By applying a term in the hopping operator of the $t-J$ model to one of such longer string states which are components of $\left|\Psi_{\langle i, j\rangle}\right\rangle$ we can create a string state which is a component of $\left|\Psi_{\langle j, n\rangle}\right\rangle$. Thus we see that the above-discussed process during which a bare hole pair has been shifted from the pair of sites $i, j$ to the pair of sites $j, n$ and which is represented by the sequence of diagrams, Fig. 1a, c, d, is an example of a whole class of analogous processes 
involving many different pairs of string states. In the same way as described above an SBP may move between all bonds which share one end point. Thus, the appropriate contribution to an effective Hamiltonian $H_{\text {eff }}$ defined in terms of operators $b_{i, j}^{\dagger}$ and $b_{i, j}$ which create and annihilate SBPs at links between NN sites $i, j$ is

$$
H_{\mathrm{eff}}^{(1)}=\tau_{1} \sum_{\langle i, j, m\rangle} b_{i, j}^{\dagger} b_{j, m} .
$$

The operators $b_{i, j}^{\dagger}$ and $b_{i, j}$ transform the ground state $|\Omega\rangle$ of the Heisenberg model on the honeycomb lattice into the wave function (1) of the SBP created at the sites $i, j$ and vice versa, respectively. $\Omega$ plays in our description the role of the vacuum state. The summation in (4) is carried over all sets containing three different sites $\langle i, j, m\rangle$, the first and the third of which are $\mathrm{NN}$ of the second. The order of indices $i$ and $m$ in the sum is relevant. Since no more than one SBP which corresponds to a fermion pair can be created at a given pair of NN sites, operators $b$ obey the hard-core constraint. In addition, it follows from the construction of SBPs that two of them cannot occupy two different links which end in the same site, which means that the effective Hamiltonian which we will derive actually belongs to the class of dimer models. The latter constraint that additionally restricts the Hilbert space in which $H_{\text {eff }}$ is defined also originates with the Pauli principle and follows from the fact that an SBP created at the link between NN sites $i$ and $j$ has its origin in the state in which two holes have been created at this pair of sites.

The hopping integral $\tau_{1}$ in $H_{\text {eff }}^{(1)}$ is

$$
\tau_{1}=-t \sum_{\mu=1}(z-1)^{\mu-1} \alpha_{0, \mu} \alpha_{0, \mu-1}
$$

We easily recognize the first term in the sum which defines $\tau_{1}$ as a product of the bare hole-hopping integral $t$ and the prefactors $\alpha_{0,1}$ and $\alpha_{0,0}$. States depicted by Fig. 1c and d appear with those prefactors in the sums (1) representing bipolarons created at bonds $i-j$ and $j-n$. Thus the first term in the sum (5) originates with the above discussed process represented by the sequence of diagrams, Fig. 1a, c, d. The rest of terms stems from similar processes which involve longer strings. The overall sign which appears in the formula (5) has the origin in the convention which defines the form and the phase of components contributing to the wave function of the SBP (1). That sign has been determined by the form of quadratic fermionic operators creating in the AF background the bare hole pair on NN sites and by the form of operators applied to shift holes between NN sites during the construction of string states.

The form of the single-particle ground state of $H_{\text {eff }}$ should give us some information about the form of the two-hole bound state, which as we expect, will be generated on the honeycomb lattice in the $t-J$ model supplemented by some isotropic short-range interaction term. The Hamiltonian (4) can be easily diagonalized by means of the Fourier transformation. The lowest band obtained in 
this way is dispersionless, which means that the analysis of the simplest process represented by the sequence of diagrams, Fig. 1a, c, d is not sufficient to find out what is the form of the two-hole bound state in the $t-J$ model on the honeycomb lattice. At $\boldsymbol{k}=(0,0)$ the ground state of (4) is doubly degenerate and transforms according to the irreducible representation $E$ of the point group $C_{3 v}$ for the honeycomb lattice, with a fixed point at a given site. After analyzing higher order processes, one can show that the unique ground state of the full effective model is formed at $\boldsymbol{k}=(0,0)$ and that it also transforms according to the irreducible representation $E$. That lengthy analysis which has been presented elsewhere [26] will be skipped here due to the shortage of space.

\section{Discussion}

The representation $E$ according to which a bound state in the weakly doped $\mathrm{AF}$ on the honeycomb lattice is expected to transform is two-dimensional. $E$ is realized by functions $x$ and $y$ and thus the name of $p$-wave symmetry can be applied to it. The terminology of $p$-wave state may misleadingly suggest that the bound two-hole state represented by the single SBP state can have a triplet structure which is impossible because the honeycomb lattice lacks the inversion symmetry. A triplet paired state can be formed only in centrosymmetric systems because two spin $1 / 2$ fermions can create a triplet only if their wave function is even with respect to the exchange of their spin quantum numbers. Since the wave function is odd with respect to the exchange of all quantum numbers, in the case of a triplet state it should have the well-defined odd parity with respect to the exchange of their positions or actually in the case of a Cooper pair with zero momentum with respect to the sign change of opposite momenta which are possessed by fermions forming the pair. Since in a non-centrosymmetric system, parity with respect to the sign change is not a good quantum number a clean triplet cannot appear. It also follows from the above analysis that if fermions are labeled by some additional quantum numbers or flavors, these flavors cannot depend on spin if a triplet is formed, because otherwise the wave function cannot have the well-defined even parity with respect to exchange of spins only.

In conclusion, we have shown that a bound state formed by two electrons or equivalently by two holes doped to the half-filled $t-J$ model on the honeycomb lattice has $p$-wave symmetry. When the bound state condenses a paired state will be formed. It will be a mixture of a singlet and a triplet which may be attributed to the fact that the honeycomb lattice is not centrosymmetric. If the exchange interaction in the $t-J$ model is supplemented by some effective short-range isotropic attractive interaction, for example induced by phonons, these conclusions are also valid. The minimal value of the ratio $J / t$ for which binding starts to take place will be lowered by the presence of this interaction. If charge ordering, which effectively gives rise to freezing out of charge and spin fluctuations on every third cobalt atom, takes place in the superconducting layered cobalt oxide at the doping level $1 / 3$, the scenario presented here may be relevant to the system. 


\section{Acknowledgments}

We thank J. Spałek for fruitful discussions.

\section{References}

[1] K. Takada, H. Sakurai, E. Takayama-Muromachi, F. Izumi, R.A. Dilanian, T. Sasaki, Nature 422, 53 (2003).

[2] T. Waki, Ch. Michioka, M. Kato, K. Yohimura, K. Takada, E. Takayama-Muromachi, T. Sasaki, cond-mat/0306036.

[3] W. Higemoto, K. Ohishi, A. Koda, S.R. Saha, R. Kadono, K. Ishida, K. Takada, H. Sakurai, E. Takayama-Muromachi, T. Sasaki, Phys. Rev. B 70, 134508 (2004).

[4] M.M. Maśka, M. Mierzejewski, B. Andrzejewski, M.L. Foo, R.J. Cava, T. Klimczuk, Phys. Rev. B 70, 144516 (2004).

[5] M. Kato, C. Michioka, T. Waki, Y. Itoh, K. Yoshimura, K. Ishida, H. Sakurai, E. Takayama-Muromachi, K. Takada, T. Sasaki, J. Phys., Condens. Matter 18, 669 (2006).

[6] Y. Kobayashi, M. Yokoi, M. Sato, J. Phys. Soc. Jpn. 72, 2453 (2003).

[7] G.Q. Zheng, K. Matano, D.P. Chen, C.T. Lin, Phys. Rev. B 73, 180503(R) (2006).

[8] T. Fujimoto, G.Q. Zheng, Y. Kitaoka, R.L. Meng, J. Cmaidalka, C.W. Chu, Phys. Rev. Lett. 92, 047004 (2004).

[9] G.Q. Zheng, K. Matano, R.L. Meng, J. Cmaidalka, C.W. Chu, J. Phys., Condens. Matter 18, L63 (2006).

[10] G. Baskaran, Phys. Rev. Lett. 91, 097003 (2003).

[11] A. Tanaka, X. Hu, Phys. Rev. Lett. 91, 257006 (2003).

[12] B. Kumar, B.S. Shastry, Phys. Rev. B 68, 104508 (2003).

[13] C. Honerkamp, Phys. Rev. B 68, 104510 (2003).

[14] Q.H. Wang, D.H. Lee, P.A. Lee, Phys. Rev. B 69, 092504 (2004).

[15] Y. Tanaka, Y. Yanase, M. Ogata, J. Phys. Soc. Jpn. 73, 319 (2004).

[16] H. Ikeda, Y. Nishikawa, K. Yamada, J. Phys. Soc. Jpn. 73, 17 (2004).

[17] K. Kuroki, Y. Tanaka, R. Arita, Phys. Rev. Lett. 93, 077001 (2004).

[18] M.D. Johannes, I.I. Mazin, D.J. Singh, D.A. Papaconstantopoulos, Phys. Rev. Lett. 93, 097005 (2004).

[19] K. Kuroki, Y. Tanaka, R. Arita, Phys. Rev. B 71, 024506 (2005).

[20] M. Vojta, E. Dagotto, Phys. Rev. B 59, R713 (1999).

[21] O.I. Motrunich, P.A. Lee, Phys. Rev. B 69, 214516 (2004).

[22] K.W. Lee, J. Kunes, W.E. Pickett, Phys. Rev. B 70, 045104 (2004).

[23] W. Zheng, J. Oitmaa, C.J. Hamer, R.R.P. Singh, Phys. Rev. B 70, 020504(R) (2004).

[24] H. Watanabe, M. Ogata, J. Phys. Soc. Jpn. 74, 2901 (2005).

[25] D. Qian, D. Hsieh, L. Wray, Y.-D. Chuang, A. Fedorov, D. Wu, J.L. Luo, N.L. Wang, L. Viciu, R.J. Cava, M.Z. Hasan, Phys. Rev. Lett. 96, 216405 (2006).

[26] P. Wróbel, W. Suleja, Phys. Rev. B 75, 174522 (2007). 